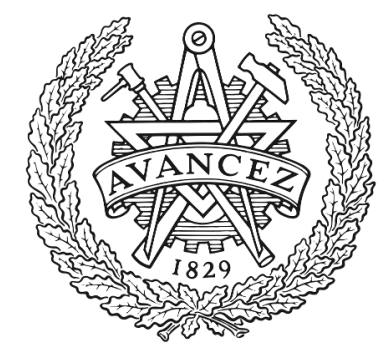

CHALMERS

UNIVERSITY OF TECHNOLOGY

\title{
Laser Frequency Combs for Coherent Optical Communications
}

Downloaded from: https://research.chalmers.se, 2023-04-26 08:47 UTC

Citation for the original published paper (version of record):

Torres Company, V., Fülöp, A. (2018). Laser Frequency Combs for Coherent Optical Communications. European Conference on Optical Communication, ECOC, 2018-September. http://dx.doi.org/10.1109/ECOC.2018.8535520

N.B. When citing this work, cite the original published paper. 


\title{
Laser Frequency Combs for Coherent Optical Communications
}

\author{
Victor Torres-Company and Attila Fülöp \\ Department of Microtechnology and Nanoscience, Chalmers University of Technology, SE-41296 \\ Gothenburg, Sweden. E-mail: torresv@chalmers.se
}

\begin{abstract}
Frequency combs can replace multiple lasers in WDM systems. We highlight performance requirements for frequency combs in coherent communications and present recent results using Kerr combs for multi-Tb/s transmission using advanced modulation formats.
\end{abstract}

\section{Introduction}

The spectrum of a laser frequency comb consists of multiple and equidistant optical frequencies that maintain a fixed phase relation. The absolute location of the lines is set by two frequencies i.e., the repetition rate, $\mathrm{f}_{\mathrm{r}}$, which defines the spacing between consecutive comb lines, and the offset, $f_{0}$, which defines the frequency shift of the comb with respect to an ideal ruler of frequencies evenly spaced by the repetition rate.

Key developments during the late $90 \mathrm{~s}$ facilitated phase locking these two frequencies to atomic radio-frequency references, thereby establishing a coherent and bi-directional link between the optical and microwave domains. This allowed to operate the frequency comb as an optical synthesizer, whereby virtually any frequency within the laser spectrum could be generated with the accuracy and precision provided by electronics. Vice versa, a laser frequency comb provides the optical clockwork necessary to count the cycles of optical frequency standards and establish a comparison with radio-frequency references. The core of a laser frequency comb is typically a mode-locked laser delivering ultrafast optical pulses at a rate commensurate to the free spectral range of the cavity. The cavity length and gain elements are actuated to stabilize the two degrees of freedom mentioned above.

Some of the key enabling features of a laser frequency comb are: (i) multiple frequencies are simultaneously available from a single laser; (ii) the line spacing is equidistant and can be locked to a stable microwave frequency; (iii) phase coherence is maintained across the bandwidth, and (iv) the optical lines can be known with a relative uncertainty to the $10^{-17}$ level ${ }^{1}$.

Optical frequency combs also offer unique opportunities in fiber-optic communication systems. In wavelength division multiplexing (WDM) for example, see Fig. 1, a single frequency comb could replace hundreds of lasers $^{2-4}$ both at the transmitter and receiver side $^{5}$. The tight frequency locking between lines alleviates the need for wavelength control at the individual channel level and enables a dramatic increase in spectral efficiency by eliminating the channel guardbands ${ }^{6}$. In addition, frequency locking has proven key to mitigate inter-channel nonlinear cross-talk by digital pre-compensation ${ }^{7}$. Another exciting aspect of laser frequency combs is the possibility to exploit the broadband phase coherence among channels to simplify the digital signal processing chain at the receiver side by performing joint impairment compensation ${ }^{8}$. These aspects are enabled by the unique features of frequency combs and are difficult, if not impossible, to implement with an array of individual lasers.

The requirements of a laser frequency comb in WDM systems are very different to the requirements for other metrology applications. Absolute accuracy with 17 digits of precision is not needed but the comb's repetition rate must be equal to the channel spacing, which is in the order of $10-100 \mathrm{GHz}$ and therefore difficult to attain with standard mode-locked lasers. In addition, for practical purposes, a WDM transceiver should be integrated on a photonic chip. The need for an integrated comb to light simultaneously hundreds of WDM channels raises concerns with regards to the minimum optical signal to noise ratio (OSNR) and power per line that can be obtained. The purpose of this contribution is to revisit quantitative benchmarks for coherent communications that illustrate some of the challenges involved. We will highlight our recent results showing multi-Tb/s data transmission using high-order complex modulation formats (PM 64-QAM) encoded onto the lines of a Kerr microresonator comb ${ }^{9}$. These results indicate these integrated comb sources are reaching a significant maturity level and may become serious contenders for practical applications in WDM.

\section{Performance requirements of laser frequency combs in WDM systems}

Let us consider a WDM system like the one sketched in Fig. 1, where a single polarization is depicted for simplicity. At the transmitter side, the individual channels are given by the frequency lines of an optical frequency comb. The lines are 

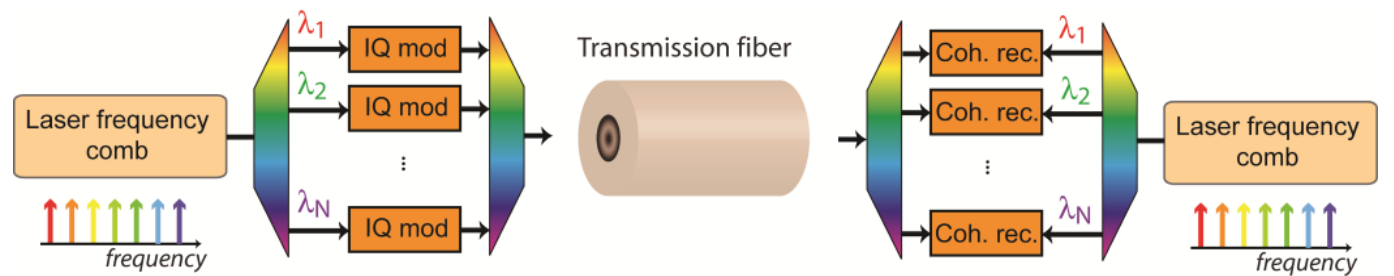

Fig. 1: Schematic representation of a WDM transceiver where the multi-wavelength laser array at both transmitter and receiver sides are implemented by a laser frequency comb.

modulated in amplitude and phase, and subsequently multiplexed to generate the signal transmitted through the fiber link. At the receiver side, the channels are demultiplexed. Each WDM channel is measured with the aid of a coherent receiver (only hardware components depicted for simplicity). The required local oscillator lasers are replaced by another frequency comb, whose line spacing and offset are matched to the transmitter side's comb.

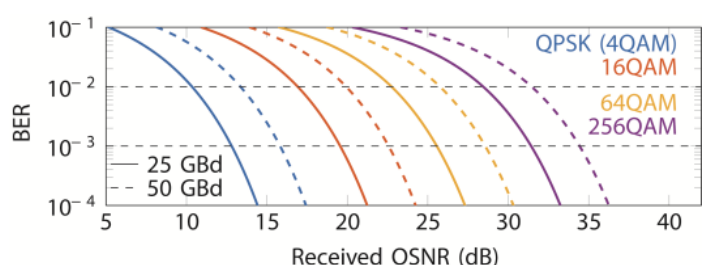

Fig. 2: Bit error ratio as function of received optical signal to noise ratio $(0.1 \mathrm{~nm}$ bandwidth) for different modulation formats and symbol rates. Simulations assume Gray coding and additive white Gaussian noise.

External cavity tunable lasers typically have optical powers in the order of $100 \mathrm{~mW}$, instantaneous linewidths below $100 \mathrm{kHz}$ and side-mode supression ratios $>50 \mathrm{~dB}$. The optical power and signal to noise ratio are not the main limiting factor when used as WDM sources. The situation is different when the WDM channels are provided by an optical frequency comb. Figure 2 shows the achievable bit error ratio (BER) when varying OSNR for different modulation formats and symbol rates. It is interesting to note that doubling the baud rate or increasing 2 bits per symbol requires $3 \mathrm{~dB}$ extra in received OSNR at a BER threshold of $10^{-3}$. The examples are better illustrated in Table 1, where the minimum required OSNR is supplemented with the linewidth requirements. Essentially, OSNRs $\sim 30$ $40 \mathrm{~dB}$ per line and $\sim 100 \mathrm{kHz}$ linewidth are needed for high-order (64-QAM and beyond) modulation formats. This performance level can be reached with bulky electro-optic frequency comb generators but it is challenging to attain with an integrated comb source that is aimed to cover a broad bandwidth and large number of lines.

\section{PM-64QAM experiments with microresonator frequency combs}

Over the last decade, there has been tremendous progress in integrated frequency comb sources in the $1.5 \mu \mathrm{m}$ window. A very promising type of integrated frequency comb are microresonator (or Kerr) frequency combs. The comb is generated by tuning a single frequency laser into the longitudinal mode of a high-Q microcavity, building up the power and allowing for the generation of a multitude of frequencies via fourwave mixing and soliton generation ${ }^{10}$. The attractiveness of microresonator combs for optical communications is that the line spacing is defined by the free spectral range of the cavity, which can easily reach $100 \mathrm{GHz}$ for millimeter long cavities, and the fabrication techniques are compatible with silicon photonics using e.g. silicon nitride microresonators. More importantly, the bandwidth can be accurately controlled by engineering the dispersion of the cavity. Recent experiments have demonstrated advanced coherent communications with WDM carriers provided by a temporal cavity soliton generated in a silicon nitride microresonator comb covering the $\mathrm{C} \& \mathrm{~L}$ bands with $100 \mathrm{GHz}$ spacing $^{5}$. These experiments nicely illustrated that the inherent parallelism of frequency combs could be exploited at the transmitter and receiver sides.

Tab. 1: Minimum requirements per wavelength and polarization channel for different modulation formats. The linewidth refers to the maximum instantaneous bandwidth that can be tolerated before an OSNR penalty of $0.2 \mathrm{~dB}$ is incurred. The OSNR at the transmitter is calculated from Fig. 2 for a BER threshold of $10^{-3}$. The values here include a more realistic transceiver with 5 ENOBs $^{11}$.

\begin{tabular}{cccc}
$\begin{array}{c}\text { Modulation } \\
\text { format }\end{array}$ & $\begin{array}{c}\text { Symbol rate } \\
\text { (GBd) }\end{array}$ & $\begin{array}{c}\text { Linewidth } \\
(\mathbf{k H z})\end{array}$ & $\begin{array}{c}\text { Tx OSNR (dB) } \\
\text { @ 0.1 nm RBW }\end{array}$ \\
\hline 16 QAM & 25 & 430 & 20.6 \\
& 50 & 860 & 23.6 \\
& 100 & 1720 & 26.6 \\
\hline 64 QAM & 25 & 125 & 27.1 \\
& 50 & 250 & 30.1 \\
- & 100 & 500 & 33.1 \\
\hline 256 QAM & 25 & 37.5 & 34.4 \\
& 50 & 75 & 37.4 \\
& 100 & 150 & 40.4 \\
\hline
\end{tabular}

We have recently investigated the use of mode-locked dark pulses in silicon nitride microresonators ${ }^{12}$ for coherent optical communications $^{9}$. These dark pulses are different to temporal cavity solitons in that they operate in the normal dispersion regime and 

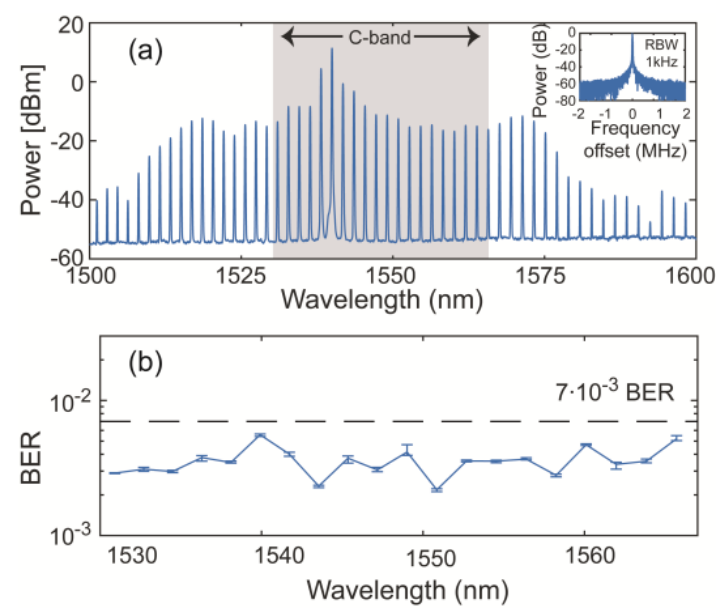

Fig. 3: (a) Optical spectrum of a $230 \mathrm{GHz}$ microresonator comb. Inset denotes the line spacing stability measured by electro-optic downconversion. (b) Achieved BER upon propagation in $80 \mathrm{~km}$ single-mode fiber when encoding $20 \mathrm{GBd}$ PM-64QAM data onto individual the comb lines within the $\mathrm{C}$ band ${ }^{9}$.

display much higher pump to comb power conversion efficiency. This is an important characteristic in fiber-optic coherent communications because it allows to achieve high OSNRs while maintaining a laser pump power commensurate to the levels provided by hybrid silicon lasers. Figure 3 shows the optical spectrum of the microresonator comb with an onchip coupled power of $\sim 20 \mathrm{dBm}$. The inset is the measured RF beat between two consecutive lines, indicating mode-locking operation and a stability level beyond what could be obtained with independent standard tunable lasers. With conversion efficiencies in the order of $20 \%$, we achieved WDM signals with an OSNR of $33 \mathrm{~dB}$ after channel equalization and amplification. The linewidth of the comb lines was measured below $10 \mathrm{kHz}$. Altogether, such performance allowed us to successfully encode polarization multiplexed 64 QAM onto all the comb lines (see Fig. 3), yielding an aggregate data rate of 4.4 . Tb/s. These results represent the highest modulation complexity ever encoded on an integrated frequency comb source.

\section{Conclusions}

Laser frequency combs provide unique opportunities in fiber-optic communication systems. The broadband phase coherence, frequency spacing stability and multitude of lines from a single laser source enable system-level performance enhancements beyond what is possible with an array of independent tunable lasers. Notwithstanding, practical WDM systems introduce stringent constraints with regards to OSNR and linewidth that are difficult to attain with integrated laser frequency combs. Microresonator combs offer an opportunity to achieve
WDM channels with a sufficient OSNR to encode complex modulation formats and yet keep a pump power level compatible with silicon photonic hybrid lasers.

\section{Acknowledgements}

We acknowledge fruitful discussions with our colleagues Mikael Mazur, Jochen Schröder and Peter Andrekson at Chalmers University of Technology. The experiments highlighted in Fig. 3 were realized with microresonator combs kindly provided by the groups of Andrew M. Weiner and Minghao Qi at Purdue University. This work has received financial support from the Swedish Research Council and the European Research Council (ERC-CoG DarkComb, grant agreement 771410).

\section{References}

[1] D. R. Carlson et al., "An ultrafast electro-optic light source with sub-cycle precision," arXiv:1711.08429 (2017)

[2] T. Ohara et al., "Over-1000-channel ultradense WDM transmission supercontinuum multicarrier source," J. Lightwave Technol., Vol. 24, No. 6, p. 2311 (2006).

[3] V. Ataie et al., "Ultrahigh count coherent WDM channels transmission using optical parametric comb-based frequency synthesizer," J. Lightwave Technol., Vol. 33, No. 3, p. 694 (2015).

[4] B. J. Puttnam et al., " $2.15 \mathrm{~Pb} / \mathrm{s}$ transmission using a 22 core homogeneous single-mode multi-core fiber and wideband optical comb," Proc. ECOC, PDP, Valencia (2015). DOI: 10.1109/ECOC.2015.7341685.

[5] P. Marin-Palomo et al., "Microresonator-based solitons for massively parallel coherent optical communications," Nature, Vol. 546, June, p. 274 (2017).

[6] M. Mazur et al., "High spectral efficiency PM-128 QAM comb-based superchannel transmission enabled by a single shared optical pilot tone," J. Lightwave Technol., Vol. 36, No. 6, p. 1318 (2018).

[7] E. Temprana et al., "Overcoming Kerr-induced capacity limit in optical fiber transmission," Science, Vol. 348, No. 6242, p. 1445 (2015).

[8] L. Lundberg et al., "Joint carrier recovery for DSP complexity reduction in frequency comb-based superchannel transceivers," Proc. ECOC, paper Th1D, Gothenburg (2017).

[9] A. Fülöp et al., "High-order coherent communications using mode-locked dark-pulse Kerr combs from microresonators," Nature Comm., Vol. 9, No. 1598, p. 1, (2018). DOI: DOI: 10.1038/s41467-018-04046-6.

[10]A. Pasquazzi et al., "Micro-combs: a novel generation of optical sources," Phys. Rep., Vol. 729, January, p. 1 (2018).

[11]T. Pfau et al., "Hardware-efficient coherent digital receiver concept with feedforward carrier recovery for M-QAM constellations," J. Lightwave Technol., Vol. 27, No. 8, p. 989 (2009)

[12]X. Xue et al., "Mode-locked dark pulse Kerr combs in normal-dispersion microresonators," Nature Photon., Vol. 9, No. 9, p. 594 (2015). 